\title{
Evaluation of the necessity for chest drain placement following thoracoscopic wedge resection
}

\author{
Ting-Yu Lu ${ }^{1} \cdot$ Jian-Xun Chen ${ }^{1}$ Pin-Ru Chen ${ }^{1}$ Yu-Sen Lin ${ }^{1} \cdot$ Chien-Kuang Chen ${ }^{1}$. \\ Pei-Yu Kao ${ }^{1} \cdot$ Tzu-Ming Huang ${ }^{1} \cdot$ Hsin-Yuan Fang ${ }^{1}$
}

Received: 27 March 2016 / Accepted: 9 August 2016 / Published online: 29 September 2016

(C) The Author(s) 2016. This article is published with open access at Springerlink.com

\begin{abstract}
Purpose To evaluate the outcomes of patients who underwent thoracoscopic wedge resection without chest drain placement.

Methods The subjects of this retrospective study were 89 patients, who underwent thoracoscopic wedge resection at our hospital between January, 2013 and July, 2015. A total of 45 patients whose underlying condition did not meet the following criteria were assigned to the "chest drain placement group" (group A): peripheral lesions, healthy lung parenchyma, no intraoperative air leaks, hemorrhage or effusion accumulation, and no pleural adhesion. The other 44 patients whose underlying condition met the criteria were assigned to the "no chest drain placement group" (group B). Patient characteristics, specimen data, and postoperative conditions were analyzed and compared between the groups.

Results Group A patients had poorer forced expiratory volume in one second (FEV1) values, less normal spirometric results, significantly higher resected lung volume, a greater maximum tumor-pleura distance, and a larger maximum tumor size. They also had a longer postoperative hospital stay. There was no difference between the two groups in postoperative complications.

Conclusions Avoiding chest drain placement after a thoracoscopic wedge resection appears to be safe and beneficial for patients who have small peripheral lesions and healthy lung parenchyma.
\end{abstract}

Hsin-Yuan Fang

seawindss@hotmail.com

1 Division of Thoracic Surgery, Department of Surgery, China Medical University Hospital, No. 2, Yude Rd., North Dist., 404 Taichung City, Taiwan
Keywords Chest tubes $\cdot$ Drainage $\cdot$ Pulmonary surgical procedure · Video-assisted thoracoscopic surgery · Pulmonary surgical procedure

\section{Introduction}

Traditionally, a chest drain is left in the pleural cavity after thoracoscopic wedge resection of the lung to mitigate against possible hemorrhage and air or lymphatic leakage $[1,2]$. However, placing a chest drain is associated with pain, immobilization, an increased risk of wound infection, and poor healing. These adverse effects may cause postoperative complications and prolong the hospital stay [3-6]. Minimally invasive thoracoscopic wedge resection of the lung has been performed routinely at our institution over the last 5 years. Unless a patient had emphysematous changes of the lung or an incorrect staple application during a lung parenchyma resection, air leaks from the staple line were uncommon. We conducted this retrospective study to evaluate the outcomes of patients who underwent thoracoscopic wedge resection without chest drain placement.

\section{Methods}

\section{Study design}

All operations were conducted at the China Medical University Hospital. Patients who underwent elective thoracoscopic wedge resection and who provided informed consent were included in this study, whereas patients who underwent mechanical pleurodesis for a pneumothorax, radical lymph node dissection for lung cancer, or bilateral lung surgery at the same time were excluded. Between 
January, 2013 and July, 2015, 89 patients who underwent thoracoscopic wedge resection for metastatic pulmonary nodules $(n=39)$, infected pulmonary lesions $(n=18)$, primary lung cancer $(n=18)$, and benign pulmonary nodules $(n=14)$ were enrolled in this study. No chest drain placement was considered for patients whose condition met all the following criteria: the lesion was located in the outer third of the lung field; there were no bullous or emphysematous changes in the lung; there were no air leaks, seen grossly or during a water sealing test; there was no dense adhesion of pleura; and there was no oozing or accumulation of pleural effusion. If any criteria were not met, a chest drain was placed intraoperatively. Forty-five patients whose condition did not meet the criteria were assigned to the "chest drain placement group" (group A), and 44 patients whose condition met the criteria were assigned to the "no chest drain placement group" (group B). Patient characteristics, specimen data, and postoperative conditions were analyzed and compared between the groups.

\section{Operative management}

All patients underwent thoracoscopic wedge resection of the lung via the 4th, 6th, and 8th intercostal spaces, using the three ports technique with $10 \mathrm{~mm}$ ports, under general anesthesia with single lung ventilation. A lung parenchyma resection was performed with ECHELON FLEX ${ }^{\mathrm{TM}}$ ENDOPATH $^{\circledR}$ Staplers (3.5/4.1 mm staple height, Ethicon, US). In group A, a \#16 French SKATER drain (Angiotech, USA) was placed via the 6th intercostal port. In group B, air evacuation was achieved by temporarily inserting a nasogastric (NG) tube into the pleural cavity, with a bowl of aseptic water as the water seal system (Fig. 1). When no air leaked while the lung was inflated (maximum airway pressure of $25 \mathrm{~mm} \mathrm{Hg}$ ), the NG tube was removed, and the residual wound was immediately closed with subcutaneous sutures.

\section{Postoperative management}

Patients were not allowed any oral intake for $4 \mathrm{~h}$ postoperatively, after which oral pain control was given, as acetaminophen $500 \mathrm{mg}$, four times a day. An intramuscular opioid (meperidine $30 \mathrm{mg}$, with a minimum $6 \mathrm{~h}$ interval) was provided for patients with intolerable pain. Chest radiographs were arranged just after surgery. If there were no abnormal findings or clinical symptoms, additional chest radiographs were obtained on postoperative days 2 and 4, and then on the day of discharge for both groups. The chest drain was removed when the amount of drainage was $<100 \mathrm{ml} /$ day and there was no evidence of ongoing bleeding or air leakage. Patients were not discharged until at least $6 \mathrm{~h}$ after drain removal.

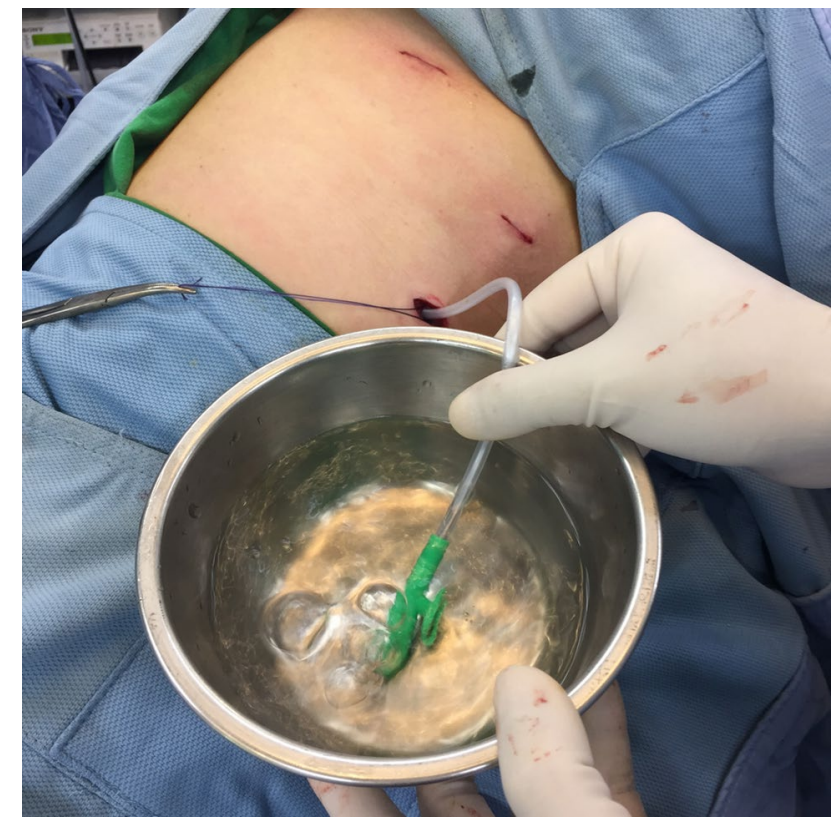

Fig. 1 In group B (no chest drain), air evacuation was achieved by temporarily inserting a nasogastric (NG) tube into the pleural cavity with a bowl of aseptic water as the water seal system. The NG tube was removed when there was no air leak during lung inflation

\section{Statistical analysis}

All continuous variables were compared with the Student's $t$ test and categorical variables were compared with the Fisher's exact test. All probability values were two tailed, and $p<0.05$ was considered significant. Statistical analysis was performed using Stata 13.1 software (StataCorp, USA).

\section{Ethical statement}

This retrospective study was approved by the research ethics committee of China Medical University \& Hospital, Taichung, Taiwan (CMUH104-REC1-119).

\section{Results}

\section{Patient characteristics}

Table 1 lists the patient characteristics. There was no significant difference between the groups in terms of age, gender, and forced expiratory volume in one second (FEV1)/ forced vital capacity (FVC). However, the group A patients had significantly poorer FEV1 values $(84.36 \pm 18.05$ vs. $95.87 \pm 13.38 \%, p=0.002)$ and significantly fewer normal spirometric results (42.86 vs. $75.00 \%, p=0.004$ ) than the group B patients. Four patients in group A and three 
patients in group B did not undergo a preoperative lung function test. Six patients in group A could not complete the preoperative lung function test and were considered to have abnormal lung function.

\section{Specimen data}

Table 2 lists the specimen data. There was no difference between group A and group B in terms of the number of wedge resections and final pathology reports. However, the

Table 1 Patient characteristics

\begin{tabular}{llll}
\hline & Group A $(N=45)$ & Group B $(N=44)$ & $p$ value \\
\hline Gender & & & \\
Female & 24 & 23 & \\
$\quad$ Male & 21 & 21 & 1.000 \\
Age (years) & $56.98 \pm 16.33$ & $54.14 \pm 12.94$ & 0.366 \\
FEV1/FVC $(\%)$ & $73.22 \pm 2.95$ & $74.22 \pm 4.13$ & 0.225 \\
FEV1 (\%) & $84.36 \pm 18.05$ & $95.87 \pm 13.38$ & 0.002 \\
Spirometry & & & \\
Normal & 18 & 30 & 0.004 \\
Abnormal & 24 & 10 & \\
\hline
\end{tabular}

Group A: patients with chest drain placement; Group B: patients without chest drain placement

FEV1 forced expiratory volume in one second, $F V C$ forced vital capacity

Table 2 Specimen findings

\begin{tabular}{lllr}
\hline & Group A $(N=45)$ & Group B $(N=44)$ & $p$ value \\
\hline Resected lung volume $\left(\mathrm{cm}^{3}\right)$ & $59.02 \pm 52.84$ & $17.81 \pm 14.67$ & $<0.001$ \\
Maximum tumor-pleura distance $(\mathrm{mm})$ & $6.46 \pm 8.01$ & $3.36 \pm 5.34$ & 0.034 \\
Maximum tumor size $(\mathrm{mm})$ & $16.36 \pm 10.1$ & $9.64 \pm 5.76$ & $<0.001$ \\
Wedge number & $1.42 \pm 0.69$ & $1.2 \pm 0.46$ & 0.837 \\
Final pathology & & & \\
Infectious lesion & 9 & 9 & \\
Benign nodule & 7 & 7 & \\
Metastatic lesion & 19 & 20 & \\
Primary lung cancer & 10 & 8 & \\
\hline
\end{tabular}

Group A: patients with chest drain placement; Group B: patients without chest drain placement

Table 3 Postoperative conditions group A patients had a significantly higher resected lung volume $\left(59.02 \pm 52.84\right.$ vs. $\left.17.81 \pm 14.67 \mathrm{~cm}^{3}, p<0.001\right)$, significantly greater maximum tumor-pleura distance $(6.46 \pm 8.01$ vs. $3.36 \pm 5.36 \mathrm{~mm}, p=0.034)$, and a significantly larger maximum tumor size $(16.36 \pm 10.1$ vs. $9.64 \pm 5.76 \mathrm{~mm}, p<0.001)$ than the group B patients. The variable "maximum tumor-pleura distance" was defined as the maximum distance measured from the tumor edge to the nearest visceral pleura or fissure for all nodules in the same patient. The variable "maximum tumor size" was defined as the maximum tumor size measured by ground glass opacity and the solid part for all nodules in the same patient. The final pathology reports are also presented in Table 2.

\section{Postoperative conditions}

Table 3 summarizes the postoperative data. The group A patients had a significantly longer postoperative hospital stay than the group B patients (4.13 \pm 0.87 vs. $3.14 \pm 0.98$ days, $p<0.001)$. However, there was no difference between the groups in the number of meperidine injections and postoperative subcutaneous emphysema. Regular follow-up chest radiographs confirmed the absence of a postoperative pneumothorax in all patients from both groups.

\begin{tabular}{lllr}
\hline & Group A $(N=45)$ & Group B $(N=44)$ & $p$ value \\
\hline Postoperative hospital stay (days) & $4.13 \pm 0.87$ & $3.14 \pm 0.98$ & $<0.001$ \\
Number of meperidine injection (time) & $1.44 \pm 1.14$ & $0.98 \pm 1.21$ & 0.064 \\
Postoperative subcutaneous emphysema & & & \\
Yes & 24 & 15 & 0.088 \\
No & 21 & 29 & \\
Postoperative pneumothorax & & & 1.000 \\
Yes & 0 & 0 & \\
No & 45 & 44 & \\
\hline
\end{tabular}

Group A: patients with chest drain placement; Group B: patients without chest drain placement 


\section{Discussion}

Minimally invasive thoracoscopic techniques, which aim to decrease the wound size, reduce postoperative pain, and preserve as much lung function as possible, are being used widely in many types of thoracic surgery. The pain caused by chest tube placement has become significant [7] and previous reports have studied the consequences of early timed chest tube removal $[8,9]$. Some reports have discussed the selection criteria for patients who did not require chest tube placement following thoracoscopic lung biopsy or even wedge resection $[10,11]$. We used these criteria for selecting patients in this study. In our experience, the decision regarding chest tube placement was also dependent on the resected lung volume, tumor size, distance from the tumor to pleura, and the number of wedge resections [6].

The major source of air leaks intraoperatively included the following: a raw surface after pneumolysis, the staple line after parenchyma resection, and bullae rupture caused by positive pressure ventilation. Air leaks from the staple line will be less common if the surgeon chooses the appropriate staple for parenchyma resection [12]. Thus, intraoperative lung adhesion, emphysematous change, or bullae formation are considered to be the most important factors for predicting postoperative air leaks. There was no air leak lasting more than $24 \mathrm{~h}$ postoperatively in our group A patients. This study excluded pneumothorax patients who routinely received mechanical pleurodesis by performing pleural abrasion during surgery to prevent recurrence, as these patients would probably need chest tube placement because of the raw surface and oozing of injured pleura.

According to our retrospective data review of all 89 patients (group A and B), there was no severe postoperative pneumothorax or subcutaneous emphysema for which reinsertion of a chest tube would have been indicated. There was only minor, nonprogressive subcutaneous emphysema in 21 group A patients and 29 Group B patients. There were no mortalities during hospitalization or in the 30 days of outpatient follow-up. Postoperative pain was evaluated by comparing the number of meperidine injections needed between the two groups, although the results were controversial. This could be because we used a \#16 French drain, which is thinner than traditional chest tubes and may reduce postoperative pain. The mean duration of chest drain placement was 2.2 days in group A. There was a significant difference in the length of the postoperative hospital stay between the two groups. A shorter hospital stay promotes patients resuming their daily lives and saves on medical costs.

\section{Study limitations}

This study had several limitations. First, as these 89 patients were reviewed retrospectively, some data may not have been collected correctly or appropriately. Second, a pulmonary function test could only be obtained preoperatively; however, several patients could not complete the testing. Furthermore, postoperative pulmonary function may be an important factor when evaluating a patients' recovery. Third, the severity of any emphysema changes and bullae formation in the lung could only be assessed by chest computerized tomography and gross observation during the operation. There was no suitable quantification for parenchyma changes, and the surgeon's decisions during the operation could only be estimated based on the surgical record. Finally, although we attempted to standardize the thoracoscopic wedge resection procedure, there were variations among individuals. For example, the incision size was based on the size of the specimen. Intercostal nerve injury caused by electrocautery or instrument compression was related to the incision location and the width of the intercostal space. All these factors influence postoperative pain.

\section{Conclusions}

There were no severe complications in patients who did not have a chest drain placed following thoracoscopic wedge resection. Furthermore, the omission of chest drain placement contributed to a shorter postoperative hospital stay. Therefore, chest drain placement following a thoracoscopic wedge resection can be avoided for selected patients who have small peripheral lesions and healthy lung parenchyma, and may be safe and beneficial.

\section{Compliance with ethical standards}

Conflict of interest We declare no conflicts of interest.

Open Access This article is distributed under the terms of the Creative Commons Attribution 4.0 International License (http://creativecommons.org/licenses/by/4.0/), which permits unrestricted use, distribution, and reproduction in any medium, provided you give appropriate credit to the original author(s) and the source, provide a link to the Creative Commons license, and indicate if changes were made.

\section{References}

1. Gomez-Caro A, Roca MJ, Torres J, Cascales P, Terol E, Castaner $\mathrm{J}$, et al. Successful use of a single chest drain postlobectomy 
instead of two classical drains: a randomized study. Eur J Cardiothorac Surg. 2006;29:562-6.

2. Miyazaki T, Yamasaki N, Tsuchiya T, Matsumoto K, Hatachi G, Kitamura Y, et al. Management of unexpected intraoperative bleeding during thoracoscopic pulmonary resection: a single institutional experience. Surg Today. 2016;46(8):901-7.

3. Iwata T, Nagato K, Nakajima T, Suzuki H, Yoshida S, Yoshino I. Risk factors predictive of atrial fibrillation after lung cancer surgery. Surg Today. 2016;46(8):877-86.

4. Katayama H, Kurokawa Y, Nakamura K, Ito H, Kanemitsu Y, Masuda N, et al. Extended Clavien-Dindo classification of surgical complications: Japan Clinical Oncology Group postoperative complications criteria. Surg Today. 2016;46(6):668-85.

5. Russo L, Wiechmann RJ, Magovern JA, Szydlowski GW, Mack MJ, Naunheim KS, et al. Early chest tube removal after videoassisted thoracoscopic wedge resection of the lung. Ann Thorac Surg. 1998;66:1751-4.

6. Watanabe A, Watanabe T, Ohsawa H, Mawatari T, Ichimiya Y, Takahashi N, et al. Avoiding chest tube placement after videoassisted thoracoscopic wedge resection of the lung. Eur J Cardiothorac Surg. 2004;25:872-6.

7. Ueda K, Hayashi M, Tanaka T, Hamano K. Omitting chest tube drainage after thoracoscopic major lung resection. Eur J Cardiothorac Surg. 2013;44:225-9.
8. Gottgens KW, Siebenga J, Belgers EH, van Huijstee PJ, Bollen EC. Early removal of the chest tube after complete videoassisted thoracoscopic lobectomies. Eur J Cardiothorac Surg. 2011;39:575-8.

9. Xie HY, Xu K, Tang JX, Bian W, Ma HT, Zhao J, et al. A prospective randomized, controlled trial deems a drainage of $300 \mathrm{ml} /$ day safe before removal of the last chest drain after video-assisted thoracoscopic surgery lobectomy. Interact Cardiovasc Thorac Surg. 2015;21:200-5.

10. Koc T, Routledge T, Chambers A, Scarci M. Do patients undergoing lung biopsy need a postoperative chest drain at all? Interact Cardiovasc Thorac Surg. 2010;10:1022-5.

11. Nakashima S, Watanabe A, Mishina T, Obama T, Mawatari T, Higami T. Feasibility and safety of postoperative management without chest tube placement after thoracoscopic wedge resection of the lung. Surg Today. 2011;41:774-9.

12. Hashimoto A, Kuwabara M, Hirasaki Y, Tsujimoto H, Torii T, Nakamura T, et al. Reduction of air leaks in a canine model of pulmonary resection with a new staple-line buttress. J Thorac Cardiovasc Surg. 2011;142:366-71. 